\title{
Findings from a Panel Discussion on Evaluation Methods in Medical Informatics
}

\author{
Felix HOLL ${ }^{\mathrm{a}, \mathrm{b} 1}$, Marina FOTTELER ${ }^{\mathrm{a}}$, Stefan MÜLLER-MIELITZ ${ }^{\mathrm{c}}$ and \\ Walter SWOBODA ${ }^{\mathrm{a}}$ \\ ${ }^{a}$ DigiHealth Institute, Neu-Ulm University of Applied Sciences, Neu-Ulm, Germany \\ ${ }^{\mathrm{b}}$ IBE, Ludwig Maximilian University of Munich, Munich, Germany \\ ${ }^{\mathrm{c}}$ IEKF GmbH, Ibbenbüren, Germany
}

\begin{abstract}
Healthcare systems are challenged by increasing costs. Digital technology can help to combat this trend. Evaluation of these technologies is uncommon or incomplete. Scholars have called for a standardized and holistic evaluation. We provide a synthesis of an online panel on medical informatics (MI) and stipulate a discussion on new guidelines for medical informatics project evaluations. The panel consisted of presentations and a discussion. The presentations gave the participants an overview of evaluation methods currently used in different medical informatics domains and their shortcomings. The presenters highlighted new evaluation methods such as a roadmap for economic analysis of eHealth projects and the German Digital Healthcare Act methods. Participants discussed the shortcomings of RCTs and methods that need to be included in eHealth evaluation and called for new evaluation methods. The discussion showed weaknesses of the currently used methods and underlined the need for a new, holistic evaluation standard for MI.
\end{abstract}

Keywords. Evaluation Research, eHealth, Mobile Health

\section{Introduction}

Improved life expectancy, driven by new drugs, treatments, and increased multimorbidity, have led to a dramatic rise in healthcare costs in the past decades and are challenging healthcare systems globally (1). The use of digital technologies (DT) in healthcare delivery can be a means to combat the trend of increasing cost (2). The potential of DT has fueled the development of new information technologies for healthcare and increased investment in new IT solutions, and overall substantial market growth in recent years (3). The growth includes traditional medical informatics domains such as clinical information technology (IT) systems like hospital information systems, but also emerging technologies like mobile health applications and smart assistive technologies (AT) for the elderly. While these new technologies, especially using mobile devices, may increase healthcare expenditure at first, they also can improve healthcare delivery and make it more efficient and potentially reduce costs in the long run (1).

There is substantial literature for the evaluation of MI projects from theoretical textbooks to reviews (4-7). The STARE-HI provides a recommendation for evaluation results reporting (8). Despite the existing methods and literature, new technologies and

${ }^{1}$ Corresponding Author, Felix Holl, DigiHealth Institute, Neu-Ulm University of Applied Sciences, 89231 Neu-Ulm, Germany; E-mail: felix.holl@hnu.de. 
applications for health are often not evaluated systematically, and when evaluations are performed, they often not generalized and focus on individual aspects. Scholars have argued for the importance of a holistic, standardized, and comprehensive eHealth evaluation $(9,10)$. The evaluation of MI projects has gained importance through recent developments such as the Digital Healthcare Act (DVG) in Germany and the European Medical Device Regulation $(11,12)$.

This research aims to provide a synthesis of a panel about evaluation methods in medical informatics, highlights the shortcomings of the evaluation methods currently used and stipulate a discussion about new guidelines for evaluations of MI projects.

\section{Methods}

The panel had been accepted at the Medical Informatics Europe 2020 conference, but panels were canceled due to the COVID-19 pandemic. The panel was held online as part of the EFMI webinar series in July 2020 with a duration of seventy minutes and consisted of four panel presentations with 10 minutes each and a moderated expert discussion with a duration of 30 minutes. The presentations aimed to give an overview of evaluation methods currently used in different areas of MI and related fields and to make participants aware of these methods' shortcomings. The second aim of the panel presentations was to inform participants about the research currently conducted to improve medical informatics evaluation methods. The expert discussion included the panelists and the audience and aimed to identify shortcomings of current evaluation methods, identify new approaches, and formulate recommendations for different evaluation methods. Participants were asked to share experiences with evaluation shortcomings and methodologies and frameworks they have used in their work. Participants were informed about the recording before the workshop. The panel recorded, transcribed and analyzed using grounded theory (13).

\section{Results}

\subsection{Panel presentations}

The panelists' diverse backgrounds, ranging from medicine and public health to medical informatics and economics, represented medical informatics interdisciplinarity. Panelist 1, with a background in MI and public health, is researching the socioeconomic impact of AT. Panelist 2 has a background in MI and global health with experience in the implantation and evaluation of mHealth technologies in Germany and low-and-middleincome countries. He is conducting research on evaluation methods for mobile health applications. Panelist 3 is an economist with a MI certificate and the CEO of a consultancy firm for eHealth \& economics. Panelist 4 is a physician and computer scientist with 15 years of experience as a CIO of large hospitals. He is a research professor for MI at a university of applied sciences. All panelists were from Germany but previously have worked internationally in Denmark, the US, and southern African countries.

The first panelist shared the results on synthesizing the existing evidence for AT's effectiveness for the elderly, describing which types of technology are most feasible and beneficial and focus on the frailty of elderly people. Out of 11,400 records retrieved 
during the search, only 19 trials met all inclusion criteria (the most important ones being: RCT, study population $\geq 65$, no laboratory setting). Nine of those were pilot studies; five studies include caregivers, and only two considered AT's economic aspects. The second presenter gave an overview of the current state of the evaluation of mHealth applications. Key findings are that no published mHealth projects have been holistically evaluated, and most evaluations focus on individual aspects. User experience was assessed most often. No holistic evaluations were performed in published studies included in the analysis. The presenter defined holistic evaluations as including all relevant aspects in the assessment rather than looking at a single aspect. He also presented the DVG, which gives publicly insured patients to right to access digital healthcare services and the reimbursement of these services. The act includes a "fast-track" approval mechanism with a preliminary approval if an application fulfills a set of basic requirements and the obligation to prove a positive care effect (11). As an example of ongoing work on developing new evaluation methods in MI, the third panelist gave an overview of the work of the eHealth working group of the German Health Economic society (dggö) on developing a 12-point road map for the economic analysis of eHealth projects (14). The standard process allows better comparison of the results. These 12 points include all the aspects that have to be considered in an economic evaluation of eHealth applications in the opinion and consensus of the eHealth working group of the dggö. The final presenter highlighted the distinction between the evaluation of new treatments and drugs compared to the assessment of digital technologies in medicine, including a description of several additional aspects that need to be included in evaluating digital technologies. An example from the presenter's tenure as the CIO of a large university medical center concluded the presentations, where a holistic evaluation was performed before introducing a digital documentation system, in which improved job satisfaction was scored higher than increased costs and no additional medical benefit.

\subsection{Expert discussion}

The participants in the expert discussion came from the Netherlands, Malaysia, and Germany. There was consensus among the panelists and participants that the current evaluation methods used for digital solutions in medicine are inadequate and neglect several different aspects. Multiple participants stated that RCTs might not be the perfect solution. RCTs is a study design focused only on the outcome but not on additional dimensions of a digital solution. RCTs require extensive preparation and have a long duration. Moreover, RCTs are methodologically flawed for this field; blinding is challenging or impossible for eHealth interventions.

Technical aspects, such as reliability and usability, and other aspects like ethics and privacy, which are challenging to consider in an RCT, are neglected. A panelist concluded, "that RCTs should not be the standard anymore for the evaluation of telemedical and eHealth solutions." The participants agree that the evaluation should start as early as possible, ideally during the development process of new technologies and devices, and involve all relevant stakeholders. Aspects beyond the current patient-/userfocus need to be included in the evaluation. Ideally, a new study design should be created, which should be better suited to evaluating digital health solutions. Participants agreed that the development of new evaluation methods for eHealth has to be placed on the research agenda. One participant stated that "the most important aspect is that we as a scientific community have to try to establish a new, holistic evaluation standard that is broadly used and validated." 


\section{Discussion}

With examples from different MI domains, the presentations highlighted shortcomings of currently used methods and showed examples of new techniques being developed. The review of AT for the elderly showed that existing studies are not holistic enough, in addition to the lack of evidence. Also, the methods used to evaluate mHealth applications are often insufficient, including recent developments such as the methods used in the DVG. The work on the 12-point road map for eHealth projects' economic analysis showed the complexity of holistic evaluation and highlighted their need. The closing presenter's remarks gave an overview of the aspects that need to be included and highlighted the need for holistic evaluation through a real-world example. The discussion underlined these themes and the agreement that RCT studies perfectly fit the evaluation of new drugs, but they are not ideally suitable for evaluating digital health devices. The task is now to develop a new method suitable for the holistic assessment of new digital solutions. Examples from different MI domains have shown that the evaluation methods currently used often only look at individual aspects and neglect important factors. As the gold standard for evaluating medical interventions, RCTs are too complicated for the fast-changing medical informatics environment while disregarding essential elements in the evaluation. A new, holistic evaluation standard for medical informatics that is broadly used and validated should be designed and established by the scientific community, especially for quickly evolving technologies such as mHealth.

\section{References}

[1] Rodriguez Santana I, Aragón MJ, Rice N, Mason AR. Trends in and drivers of healthcare expenditure in the English NHS: a retrospective analysis. Health Econ Rev. 2020;10(1):1-11.

[2] Kannry J, Smith J, Mohan V, Levy B, Finnell J, Lehmann CU. Policy Statement on Clinical Informatics Fellowships and the Future of Informatics-Driven Medicine. Appl Clin Inform. 2020 Oct 28;11(5):710-3.

[3] Koch S. Current Trends in Biomedical and Health Informatics. Methods Inf Med. 2019 Sep 12;58(02/03):061-2.

[4] Friedman CP, Wyatt J. Evaluation methods in biomedical informatics. Hannah KJ, Ball MJ, editors. New York, NY: Springer New York; 2006. 414 p. (Health Informatics).

[5] Brender J. Handbook of Evaluation Methods for Health Informatics. Handbook of Evaluation Methods for Health Informatics. Elsevier Inc.; 2006.

[6] Eslami Andargoli A, Scheepers H, Rajendran D, Sohal A. Health information systems evaluation frameworks: A systematic review. Int J Med Inform. 2017;97:195-209.

[7] Fanta GB, Pretorius L, Erasmus L. An evaluation of ehealth systems implementation frameworks for sustainability in resource constrained environments: A literature review. IAMOT 2015 - 24th Int Assoc Manag Technol Conf Technol Innov Manag Sustain Growth, Proc. 2015;1046-63.

[8] Talmon J, Ammenwerth E, Brender J, de Keizer N, Nykänen P, Rigby M. STARE-HI-Statement on reporting of evaluation studies in Health Informatics. Int J Med Inform. 2009;78(1):1-9.

[9] Catwell L, Sheikh A. Evaluating eHealth interventions: The need for continuous systemic evaluation. PLoS Med. 2009;6(8):1-6.

[10] Enam A, Torres-Bonilla J, Eriksson H. Evidence-based evaluation of ehealth interventions: Systematic literature review. J Med Internet Res. 2018;20(11).

[11] Gerke S, Stern AD, Minssen T. Germany's digital health reforms in the COVID-19 era: lessons and opportunities for other countries. npj Digit Med. 2020;3(1):1-6.

[12] Becker K, Lipprandt M, Röhrig R, Neumuth T. Digital health - Software as a medical device in focus of the medical device regulation (MDR). it - Inf Technol. 2019 Oct 1;61(5-6):211-8.

[13] Birks M, Mills J. Grounded Theory A Pratical Guide. 2nd Editio. SAGE Publications Ltd; 2015.

[14] Köberlein-Neu J, Müller-Mielitz S. Roadmap zur Entwicklung eines Evaluationskonzeptes. In: Müller-Mielitz S, Lux T, editors. E-Health-Ökonomie. Springer Fachmedien Wiesbaden; 2017. p. 881-92. 